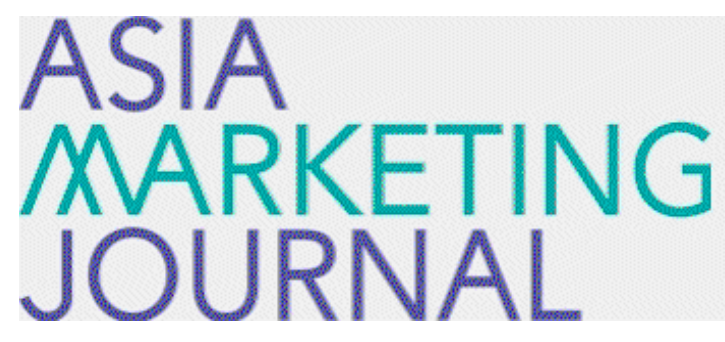

ASIA MARKETING JOURNAL

Volume 21 | Issue 4

Article 3

$1-31-2020$

\title{
The Effect of Product Experience Through Virtual Reality on Product Evaluation
}

\author{
Eunmi Jeon \\ Youngjee Han \\ Hyunjin Woo
}

Follow this and additional works at: https://amj.kma.re.kr/journal

Part of the Marketing Commons

\section{Recommended Citation}

Jeon, Eunmi; Han, Youngjee; and Woo, Hyunjin (2020) "The Effect of Product Experience Through Virtual Reality on Product Evaluation," Asia Marketing Journal: Vol. 21 : Iss. 4 , Article 3.

Available at: https://doi.org/10.15830/amj.2020.21.4.45

This Article is brought to you for free and open access by Asia Marketing Journal. It has been accepted for inclusion in Asia Marketing Journal by an authorized editor of Asia Marketing Journal. 


\title{
The Effect of Product Experience Through Virtual Reality on Product Evaluation
}

\author{
Eunmi Jeon* \\ Youngjee Han** \\ Hyunjin Woo***
}

\begin{abstract}
With the development of new technologies, virtual reality (VR) is emerging as a new consumer marketing platform. Yet, only a few studies have investigated VR in the consumption context. The current research explores how experiencing a product through VR influences consumer attitude and purchase intention for the product. Although assessing products in real life is believed to be more diagnostic than that through VR, the present research revealed that experiencing a product through VR could lead to a more positive attitude toward and higher purchase intention for the product. We also demonstrated that "enjoyment" has a mediating effect while evaluating the product through VR.

To compare product experience through VR to that in real life, we employed the HTC Vive VR system to implement a VR environment. In the VR condition, participants evaluated plastic building blocks using a VR headset and a motion controller; and in the real life (reality) condition, they evaluted plastic building blocks printed using a 3D printer to make them look exactly the same as the blocks used in the VR condition. Finally, implications and suggestions are discussed.
\end{abstract}

Keywords: Virtual Reality, Product Evaluation, Interactivity Through Consumption Experience, Purchase Intention, Telepresence, Enjoyment

\section{Introduction}

Nowadays, virtual reality (VR) is widely used, and people have become more accustomed to it. VR has been studied for a long time, and many test products have been developed. However, its commercialization has been limited due to the lack of content and high purchasing prices. Nevertheless, start-up and

\footnotetext{
* School of Business, Sungkyunkwan University (jem0510@skku.edu)

** School of Business, Sungkyunkwan University (yjhan@skku.edu), Corresponding Author

*** School of Business, Sungkyunkwan University (hjwoo247@gmail.com)
} 
major companies have recently released VR products for shopping, education, medicine, and games and entertainment. For example, the United States retail giant Walmart has acquired the VR software development company Spatialand to introduce a new distribution channel that utilizes VR. They are planning to provide online and offline VR shopping experiences for their customers. Walmart applied for a patent in the field of VR (Bloomberg 2018) to establish a VR system. In addition, the company is planning to supply VR headsets and gloves. Using this VR equipment, customers can feel like they are shopping in an offline Walmart retail store. Without the need to visit the offline store, customers can have a shopping experience as vivid as in real life.

Therefore, the paradigm of shopping through $\mathrm{VR}$ is expected to continue developing and become the new shopping platform with the advancements in VR technology. Consequently, there is a growing need to investigate the effect of VR in marketing context. Despite the importance of VR shopping systems in marketing and consumer decision-making, previous research has rarely investigated consumer response to the consumption of VR systems.

Past research revealed that direct experience with a product has a greater impact on consumer attitudes and purchasing decisions for the product than product information provided by a company or evaluations of other consumers (Coyle and Thorson 2001). Therefore, compared to the experience of directly touching and assessing a product, experiencing the product through VR might be considered ineffective in enhancing a consumer's attitude or purchase intention for the product. However, the current research predicts that evaluating a product through VR can provide more diverse and rich experience, increasing the enjoyment of the experience. Therefore, we expect that VR could have a positive impact on attitudes or purchase intentions for a product.

Specifically, the current research predicts that the vividness and enjoyment during the shopping experience will be greater in VR environments, leading to a positive effect on purchase intention. While past research has investigated the technology of VR, it has neglected how VR affects consumer experiences and decision-making processes. Therefore, using the latest VR equipment, we examined whether VR experiences lead to different results for the same product than real-life experiences. The findings of the current research contribute to the field of consumer research by examining the potential of VR as a new consumption platform.

\section{Theoretical Background}

\subsection{Virtual Reality}

After the emergence of Oculus-Rift in 2012, 
VR equipment has been widely commercialized. Today, VR has acquired attention as a new technology in various fields. The development of such new technologies can potentially increase consumers' ability to utilize information (Keohane and Joseph 1998). Since effectively delivering information to consumers is vital for companies, using efficient media has been a major concern. In particular, the advent of the internet has made it possible to provide an efficient platform for consumers to experience and buy products without visiting an offline retail store.

However, previous research has demonstrated the limitations on interaction when consumers communicate through computers; especially, the two-dimensional (2D) graphic displays are not sufficient to deliver the relevant information (Schlosser 2003; Li, Daugherty, and Biocca 2002). As mentioned above, online shopping has rapidly developed; however, online shopping using 2D media has always been limited in terms of richness of experience. Previous research has shown that offline shopping has a more positive impact on preference, satisfaction, enjoyment, and overall evaluations than online shopping (Cronin et al. 2000; Dennis, Harris, and Sandhu 2002). According to Hansen and Jensen (2009), enjoyment through online shopping is lower than that through offline shopping.

While online shopping can reduce time and space constraints, it is still limited in the sense that consumers cannot directly experience the purchasing environment. However, the limitations of online shopping by 2D media are expected to be overcome by the development of VR technology, as VR will provide richer shopping experiences for consumers.

VR aims to make people feel like they are experiencing reality by using the computer media environment (Biocca 1992). Steuer (1992) defines VR as a simulated environment or telepresence. Thus, VR can make people feel like they are experiencing reality despite being physically distant from the object. VR is the recreation of an environment through computers, based on reality. In addition, the advances in VR equipment and technology allow us to experience the vividness of reality. Thus, the current research focuses on the traits of VR that are different from reality. In particular, the fact that VR technology allows people to experience something as if it were real is fascinating enough for people, despite the limitation of physical distances. Further, VR provides a more enjoyable experience by enabling more diverse experiences than in real life.

Telepresence enables people to experience "the presence of reality" (Steuer 1992). It is important to distinguish "reality" and "virtual reality." In other words, telepresence is the experience of presence in mediated environment rather than immediate physical environment (Steuer 1992). Telepresence makes users to be immersed in the world made by media and thus, it can make people experience the flow on virtual reality (Pelet, Ettis, and Cowart 
2017). This flow refers to the overall feeling when people experience the optimal status (Csikszentmihalyi 1975). In general, the flow has been mentioned as the key concept for researches on the interaction between human and computer. More recently, some researchers have investigated the flow through interaction in virtual reality (Jung and Pawlowski 2014; Huang and Liu 2017). From this perspective, we suggest that the flow in virtual reality can induce people to experience enjoyment because it can provide the optimal state. Thus, the current research expected that telepresence through VR would evoke interest and enjoyment in the shopping environment, positively affecting consumer attitude.

Recently developed VR devices, such as a keyboard or mouse with motion controllers or a $360^{\circ}$ head-mounted displays, provide superior experiences than that of $2 \mathrm{D}$ graphics. Thus, VR equipment enhances interaction with objects, creating an experience that is closer to reality. However, creating VR with these devices requires a very high-performance computer, such as the Geforce GTX 970, and at least 8 GB RAM. On the other hand, researchers have been unable to verify the potential of VR, because they have been limited to using basic equipment or educational software when conducting their experiments on VR. (Grantcharov et al. 2004). Therefore, this research investigated the influence of VR by using high-performance computers and recently developed VR equipment.

\subsection{Object Interactivity and Consumption Experience}

When consumers buy a product or find information online, they see two-dimensional or three-dimensional images, text, or video. These online shopping experiences are the typical way to communicate with the products or brands. According to past research (Schlosser 2003; Jiang et al. 2010), sufficient interaction with a product can positively affect a consumer's product purchase intention. In other words, object interactivity is a major factor in the consumption environment, whether offline or online. Offline shopping can have a positive influence on consumer attitude as it provides direct interaction with objects. However, a study revealed that interactions through VR enable active mind-mapping, thereby allowing richer interactions (Steuer 1992). In addition, Laurel (2016) pointed out that indirect experiences through VR, with equipment such as motion controllers, enable more natural and realistic experiences for consumers, allowing for more powerful interactions. Moreover, research has shown that VR is more effective in enhancing consumers' attitude toward an object because VR makes them feel as if the object is closer than they experience the object in reality (Lanier and Biocca 1992). On the other hand, Li, Daugherty, and Biocca (2003) mentioned that consumption through VR maximizes the visualization of a product, and it can increase 
the knowledge or attitude on the product.

The current research predicts that sophisticated VR can provide a more realistic experience than that in real life and increase interaction through immersion with the object.

Although consumers recognize that what they are experiencing is not real life, the vividness provided by VR will enhance immersion with the object. Thus, we expect that the experience through VR strengthens "enjoyment" during the shopping experience.

\subsection{Enjoyment and Purchase Intention}

Previous research on consumer behavior has shown that in consumption experiences, enjoyment has a significant effect on purchase intention (Li, Daugherty, and Biocca 2002; Martineau 1958; Brown, Pope, and Voges 2003; Tauber 1972). Accordingly, many companies continue various attempts and efforts to enable consumers to enjoy their purchasing environment. As mentioned above, the current research predicts that shopping experience through VR could enable a more enjoyable experience than that in reality, which would lead to higher purchase intention (Figure 1). Thus, we predict the following:

H1: The experience through VR (vs. Reality) would increase purchase intention on product.

H2: The experience through VR (vs. Reality) would increase enjoyment.

H3: The enjoyment through VR would increase purchase intention on product. Thus, enjoyment would mediate between $V R$ (vs. reality) and purchase intention.

\subsection{Experiment}

The experiment attempted to test the main hypothesis that consumers who participate in VR experience greater enjoyment than that in reality, resulting in a positive effect on their purchasing intentions. To implement VR, we used high-performance VR equipment. Thus, we compared the evaluations on the same

\section{〈Figure 1〉 Research Model}

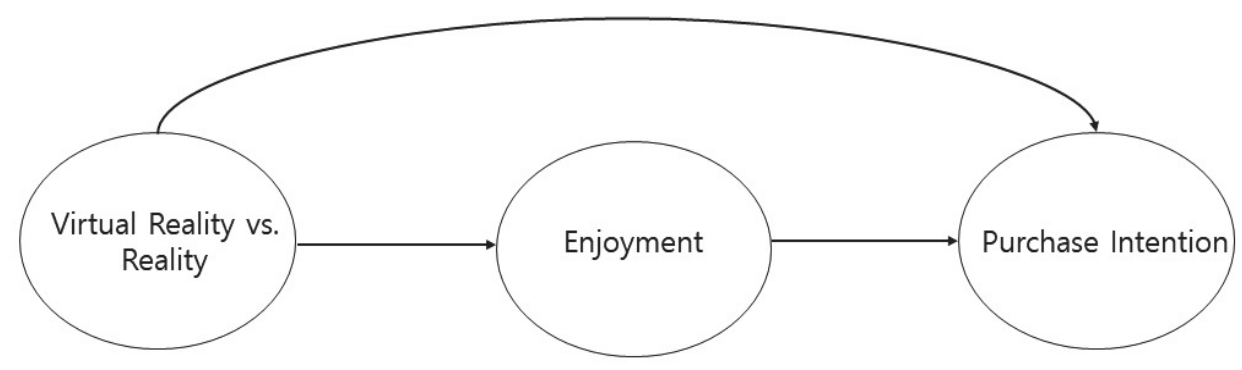

The Effect of Product Experience Through Virtual Reality on Product Evaluation 49 
product in VR and reality conditions.

\subsection{Method}

\subsubsection{Participants and Procedure}

Participants of the experiment included 32 (65.6\% male) undergraduate students who joined this experiment in exchange for extra course credit. Participants were randomly assigned to one of the two conditions ("virtual reality" versus "reality"). Participants were told that this experiment aims to investigate consumer response to building a house from a virtual/real plastic block. In the VR condition, participants were instructed to experience the block by using VR equipment. In the reality condition, participants were asked to experience the block in 3D-printed form, in the same shape as in the VR condition. In the VR condition, the HTC Vive VR system was used, along with a computer with GTX GeForce 1070 graphics, 16 GB RAM, Intel囚 CoreTM i7-7700HQ CPU $2.80 \mathrm{GHz}$ processor. Participants were explained how they could use the motion controller and head-mounted display. They were given enough time to familiarize themselves with the system. Then, they were given two different tasks. In the VR condition, they were asked to create stairs with the plastic blocks (see Appendix) on the monitor; and in the reality condition, they were asked to create stairs with actual plastic blocks. In addition, we chose plastic blocks for this experiment because product selection was limited due to technical difficulty in VR version. Afterwards, they were instructed to create a house-shaped model, freely, by using the blocks. Participants were given sufficient time for each assignment. The first task to create stairs was designed to help the participants get familiar with the environment, while the second task was designed to test our main hypothesis. After participants completed the two tasks, they were asked to respond to the questions. They were asked how willing they were to receive the plastic block free of charge and inquired about their purchase intention if the block were to be released as a product. To measure the mediation, they also rated on a seven-point scale ( $1=$ not at all, 7 = very much) how enjoyable it was to create the house with the blocks. In addition, they were asked about how novel they thought the product was and if they felt bored while evaluating it. Participants were also asked to rate on a seven-point scale ( $1=$ not at all, 7 $=$ very much) how difficult it was for them to create the house-model shape with the blocks. The time taken to complete the assignment to build a house with blocks was measured. Finally, participants provided demographic information and were thanked.

\subsubsection{Results}

To test the main hypothesis, a one-way (VR 
VS. reality condition) analysis of variance (ANOVA) was conducted with purchase intention as the dependent variable. As predicted, the ANOVA revealed that participants who evaluated the product through VR had higher intention to receive the product than those in the reality condition $\left(M_{\text {virtual reality }}=6.06\right.$ vs. $M_{\text {reality }}=5.00, F(1,30)=5.319, p=.028<$ $.05)$. The VR-condition participants indicated higher purchase intention in the future than the reality-condition participants $\left(M_{\text {virtual reality }}\right.$ $=4.31$ vs. $M_{\text {reality }}=3.13, F(1,30)=4.274$, $p=.047<.05)$. Consistent with our expectations, enjoyment in the VR condition was higher than that in the reality condition $\left(M_{\text {virtual reality }}=5.56\right.$ vs. $M_{\text {reality }}=4.56, F(1$, $30)=7.085, p=.012<.05)$. In addition, no significant differences were observed between the VR and the reality condition for the evaluation of the novelty of the product $\left(M_{\text {virtual reality }}=5.31\right.$ vs. $M_{\text {reality }}=4.44, F(1$, 30) $=2.649, p=.114$ ), feeling bored while evaluating the product $\left(M_{\text {virtual reality }}=2.69\right.$ vs. $\left.M_{\text {reality }}=2.56, F(1,30)=.083, p=.776\right)$, the effort taken to evaluate the product ( $M_{\text {virtual reality }}$ $=5.06$ vs. $M_{\text {reality }}=4.94, F(1,30)=.099$, $p=.755)$, and the time taken to completing the product $\left(M_{\text {virtual reality }}=206\right.$ seconds vs. $M_{\text {reality }}=141.13$ seconds, $F(1,30)=2.614, p$ $=.116)$.

To analyze the mediation effect of enjoyment, we followed Baron and Kenny's (1986) fourstep approach to mediation. Accordingly, we first regressed the VR (vs. reality) on participant's intention to receive the product. This analysis suggested that VR strengthened the intention to receive the product $(\beta=-1.063, p=.028$ $<$.05). The effect of VR (vs. reality) on enjoyment was significant $(\beta=-.938, p=$ $.027<.05)$. In the next step, we regressed VR (vs. reality) and participant's enjoyment on the intention to receive the product; enjoyment had a significant effect $(\beta=.622, p=.002$ $<.05$ ), while the effect of VR (vs. reality) on the intention to receive the product was not significant $(\beta=-.3479, p=.270)$. Thus, the analysis revealed that enjoyment fully mediates the relationship between the intention to receive the product and VR (vs. reality).

Second, we conducted the same analysis to confirm the mediation effect of enjoyment on purchase intention. The analysis revealed that VR had a positive effect on purchase intention $(\beta=-1.188, p=.047<.05)$. To test whether VR (vs. reality) affects enjoyment, we regressed VR on enjoyment, which revealed a positive and significant relationship between the two variables $(\beta=-.938, p=.027<.05)$. In the last step, we regressed VR (vs. reality) and enjoyment on purchase intention; enjoyment on purchase intention remained significant $(\beta=.931, p<.001)$, whereas the effect of VR (vs. reality) on purchase intention was not significant $(\beta=-.314, p=.517)$. Therefore, we can conclude that enjoyment fully mediates the relationship between VR (vs. reality) and 
〈Table 1〉 Mediation via enjoyment on purchase intention

\begin{tabular}{lcc}
\hline \multicolumn{1}{c}{ Testing steps in mediation model } & $\beta$ & $\mathrm{p}$ value \\
\hline \hline Testing Step 1 & -1.188 & .047 \\
Outcome: Purchase Intention & & \\
Predictor: VR vs. Reality & -.938 & .027 \\
Testing Step 2 & & \\
Outcome: Enjoyment & & \\
Predictor: VR vs. Reality & & \\
Testing Step 3 & .931 & .001 \\
Outcome: Purchase Intention & -.314 \\
Mediator: Enjoyment & .517 \\
Predictor: VR vs. Reality & \\
\hline
\end{tabular}

purchase intention (see table 1).

\section{General Discussion}

Today, many companies make attempts to promote their products through VR to increase purchase intention of customers. In particular, the process of evaluating a product in VR may provide a novel experience to consumers and lead to a more diverse interaction with the object, thus enabling a richer and more enjoyable experience. Therefore, VR experiences can affect consumer attitude and purchase intention. The current research used the latest VR equipment and 3D printers to provide environments similar to real-life conditions. We investigated participant attitudes on a product in the VR and the reality condition. The results revealed that VR experiences lead to higher purchase intention than real life experiences. Unlike the existing perception that shopping in real-life will have a more positive effect on consumer attitude, our findings showed that product experience through VR increases purchase intention. From this perspective, we suggest the possibility of overcoming the limitations of online shopping with VR technology.

As the purchasing environments continue to change, VR is emerging as a new marketing platform. However, there is a lack of empirical research on VR and consumer decision-making; investigation on the differences in consumer behavior between offline and online shopping environments is needed. Moreover, there is no research that compares the experiential differences in VR and real-life environments; this empirical research contributes to the field by testing two different conditions (VR versus reality) by using the latest VR equipment.

Therefore, our findings are meaningful as they revealed that VR (vs. reality) experience increases purchase intention and has a great 
potential as a consumption platform. Furthermore, the current research demonstrated the mediating effect of enjoyment on the relationship between VR (vs. reality) experience and purchase intention. Thus, we suggest that VR provides for a more effective shopping environment than reality.

These findings suggest that existing channels that provide shopping experiences through VR can overcome the spatial constraints of shopping in offline stores. In addition, it could provide a richer and more enjoyable experience than direct product experience and lead to higher purchase intention.

In this study, the same plastic blocks were used as the target product in comparing customer experiences in the VR and the reality condition. Product selection was limited due to technical difficulty of implementing the VR version. Thus, the current research showed the results using plastic blocks although VR is used for more diverse product experiences and usages. However, in the future, we expect that developments in software will allow us to investigate more various possibilities of virtual reality and consumer behavior.

We expect that different shopping environments could also influence the results. In our experiment, we asked participants to make the product by themselves, and then we asked purchase intention on the product. However, there are various consumption situations when consumers buy products through VR systems. For example, when people buy the product in online shopping, they could see the 3D product images made by VR. These indirect experiences can lead people to have different attitudes about the product. The current research did not identify the impact of VR in a more diverse consumer's environment due to equipment limitations. Therefore, the future research needs to examine the purchase behavior or evaluations through VR in various consumption environments. In addition, future research needs to control the other variable which can affect the results. For instance, usefulness of VR system may increase purchase intention of product, future research should examine the effect to rule out alternatives. Furthermore, we anticipate that VR experience may lead different results, depending on the product category. Specifically, when people consider buying the hedonic product through VR experience (vs. reality), the purchase intention would be higher than when they buy utilitarian product. Because there is the possibility that hedonic product with VR will increase enjoyment. On the other hand, when they buy the utilitarian product, the purchase intention could increase in reality shopping environment. Because buying the utilitarian product focuses more on practical value than enjoyment. Future research may explore how product category (hedonic vs. utilitarian) affects evaluation depending on shopping environments (VR vs. reality).

Although the current research revealed that 
VR experiences lead to higher purchase intention and that this effect is mediated by enjoyment, we could not show the specific traits caused by VR. It might be difficult to evaluate product attributes such as weight and texture with current VR technologies. However, VR technologies can potentially provide a richer experience, allowing consumers to focus more on the product experience. Research on VR as a new platform of consumption will expand the understanding of VR compared to reality. Furthermore, we weren't able to separate the effect of experiencing the product in VR from the effect of using the new device. Wearing the new device itself could have increased enjoyment of participants. Future research can increase our understanding of the effect of VR in consumption experience by separating the two effects. Additionally, we asked only one questions for our dependent variables and mediator. Future research can improve reliability of the results by using multiple questions for each measure.

Our findings also suggest implications for marketers who are considering using VR system. In addition, our findings have managerial implications for consumers' attitudes toward oneline shopping, marketing segmentation, improvement of oneline shopping.

We expect that the result of this research would be meaningful for marketing manager or marketing channel. Specifically, these results provide suggestions to attract consumers who prefer offline shopping to online shopping. If marketers use the VR system in various ways to provide consumers with enjoyment, the product evaluations or purchase intention will be enhanced. With the development of VR system, the technology will expand the way of shopping experiences which can provide convenience and enjoyment for consumers.

〈Received September 10. 2019〉

〈Accepted January 29. 2020〉

\section{References}

Baron, Reuben M., and David Kenny (1986), "The Moderator - mediator Variable Distinction in Social Psychological Research: Conceptual, Strategic, and Statistical Cconsiderations," Journal of Personality and Social Psychology, 51(6), 1173.

Biocca, Frank (1992), “Communication Within Virtual Reality: Creating a Space for Research," Journal of Communication, 42 (4), 5-22.

Brown, Mark, Nigel Pope, and Kevin Voges (2003), "Buying or Browsing? An Exploration of Shopping Orientations and Online Purchase Intention," European Journal of Marketing, 37(11/12), 1666-1684.

Coyle, James R., and Esther Thorson (2001), "The Effects of Progressive Levels of Interactivity and Vividness in Web 
Marketing Sites," Journal of Advertising, 30(3), 65-77.

Cronin Jr, J. Joseph, Michael K. Brady, and G.

Tomas M. Hult (2000), “Assessing the

Effects of Quality, Value, and Customer Satisfaction on Consumer Behavioral Intentions in Service Environments," Journal of retailing, 76(2), 193-218.

Csikszentmihalyi, Mihaly (1975), "Play and

Intrinsic Rewards," Journal of Humanistic Psychology, 15(3), 41-63.

Dennis, Charles, isa Harris, and Balraj Sandhu (2002), "From Bricks to Clicks: Understanding the E-consumer," Qualitative Market Research: An International Journal, 5(4), 281-290.

Grantcharov, Teodor P., Kristiansen, V. B., Bendix, J., Bardram, L., Rosenberg, J. and Funch Jensen, P. (2004), "Randomized Clinical Trial of Virtual Reality Simulation for Laparoscopic Skills Training," British Journal of Surgery, 91(2), 146-150.

Hansen, Torben, and Jan Møller Jensen (2009),

"Shopping Orientation and Online Clothing Purchases: The Role of Gender and Purchase Situation," European Journal of Marketing 43(9/10), 1154-1170.

https://www.bloomberg.com/news/articles/ 2018-08-17/walmart-eyes-virtual-realityshopping-system-patent-filings-say

Jiang, Zhenhui, Chan, J., Tan, B. C. and Chua, W. S. (2010), "Effects of Interactivity on Website Involvement and Purchase
Intention," Journal of the Association for Information Systems, 11(1), 34.

Jung, Yoonhyuk and Suzanne D. Pawlowski (2014), "Virtual goods, Real Goals: Exploring Means-End Goal Structures of Consumers in Social Virtual Worlds," Information \& Management, 51(5), 520-531.

Keohane, Robert. O. and Joseph S. Nye Jr. (1998), "Power and Interdependence in the Information Age," Foreign Affairs, 81-94.

Lanier, Jaron and Frank Biocca (1992), “An Insider's View of the Future of Virtual Reality," Journal of Communication, 42(4), 150-172.

Laurel, Brenda (2016), "What is Virtual Reality?," medium. com. Online article accessed August 9th.

Li, Hairong. Terry Daugherty and Frank Biocca (2002), "Impact of 3-D Advertising on Product Knowledge, Brand Attitude, and Purchase Intention: The Mediating Role of Presence," Journal of Advertising, 31 (3), 43-57.

Li, Hairong. Terry Daugherty and Frank Biocca (2003), "The Role of Virtual Experience in Consumer Learning," Journal of Consumer Psychology, 13(4), 395-407. Martineau, Pierre. (1958), "The Personality of the Retail Store," 47-55.

Pelet, Jean-Éric, Saïd Ettis, and Kelly Cowart (2017), "Optimal experience of flow enhanced by telepresence: Evidence from 
social media use," Information \& Management, 54(1), 115-128.

Schlosser, Ann E. (2003), "Experiencing Products in the Virtual world: The Role of Goal and Imagery in Influencing Attitudes versus Purchase Intentions," Journal of Consumer Research, 30(2), 184-198.
Steuer, Jonathan (1992), "Defining Virtual Reality: Dimensions Determining Telepresence," Journal of Communication, 42(4), 73-93. Tauber, Edward M. (1972), “Marketing Notes and Communications: Why Do People Shop?," Journal of Marketing, 36 (October), 45-59. 


\section{〈APPENDIX〉}

1. Plastic blocks $(H: 0.96 \mathrm{~cm}, L: 3.2 \mathrm{~cm}, W: 1.6 \mathrm{~cm})$ and $3 \mathrm{D}$ printer used in the experiment.
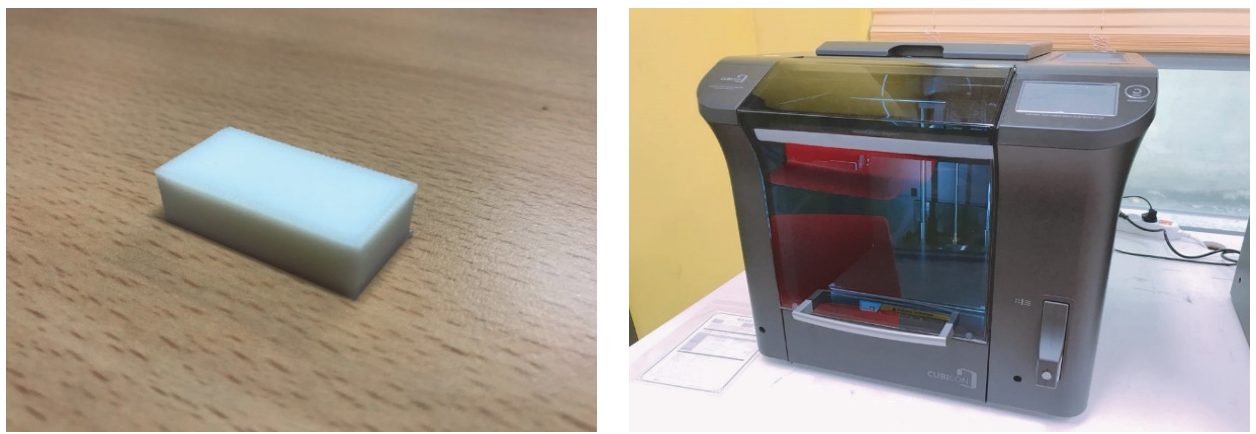

2. HTC Vive, VR equipment used in the experiment.

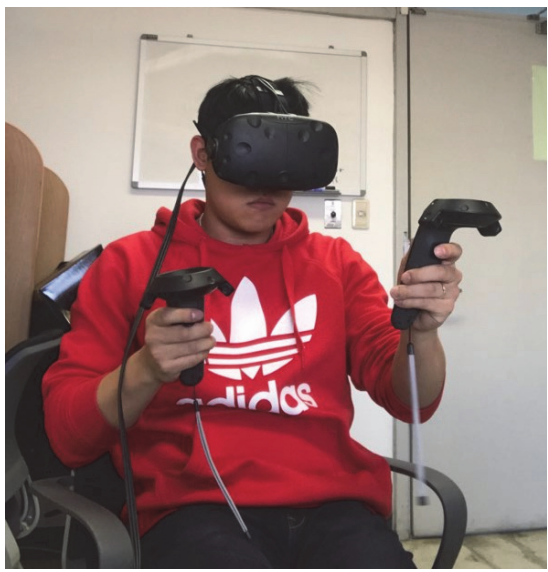

3. Example of a house built by the VR program, "Make VR Pro."

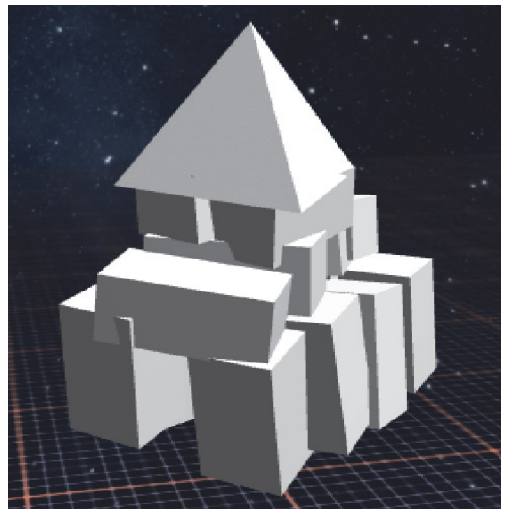

The Effect of Product Experience Through Virtual Reality on Product Evaluation 57 